\title{
Tradición y cambio en la división del trabajo comunitario: el proceso artesanal de tapetes de lana en una comunidad indígena zapoteca
}

\author{
Alejandra Elizabeth Urbiola Solis \\ Universidad Autónoma de Querétaro, México. \\ alex-urbiola@hotmail.com \\ Angel Wilhelm Vázquez García \\ Universidad Autónoma Metropolitana, Unidad Xochimilco, México. \\ avazquezg@correo.xoc.uam.mx
}

Resumen: El proceso de producción artesanal de tapetes de lana de la comunidad de Teotitlán, ubicada en la región de los Valles Centrales de Oaxaca (Oaxaca, México), se organiza con un alto grado de valoración de los elementos étnico-religiosos compartidos en la comunidad. El objetivo del trabajo fue mostrar como la elaboración de tapetes es un ejemplo de organización de energía bumana que responde a una división del trabajo tradicional donde la organización de la producción se basa en un sistema de organización del trabajo comunitario y diferenciado que asigna roles distintos a mujeres $y$ varones que son reproducidos gracias a un sistema simbólico que incluye normas de conducta. Se buscó conocer la percepción de los sujetos y las posibilidades de cambio dentro de una organización del trabajo dominada por la visión de la comunidad. Los principales resultados mostraron que la condición de emprendedor es valorada en la comunidad; sin embargo, se subsume a la condición étnica, es decir, a la reproducción explícita de la estructura social e implícita a través de la subjetividad ligada a las actividades religiosas y seculares. La división del trabajo en la producción de tapetes se organiza considerando el prestigio, los valores tradicionales y la asignación de roles por género. El éxito empresarial se entiende como un éxito de la comunidad por cuanto debe existir un equilibrio entre el uso de los recursos y la producción de tapetes por encima del éxito individual o de un solo empresario.

Palabras clave: división del trabajo, empresas familiares, comunidad, etnicidad. 


\begin{abstract}
The process of producing handmade woolen rugs from Teotitlan community, located in the region of the Central Valley of Oaxaca, Oaxaca, Mexico is organized with a high degree of value to the ethno-religious elements shared in the community. The objective of this paper was to show how the production of carpets is an example of organization of human energy that responds to a traditional division of labor where the organization of production is based on a system of organization of community work and differentiated that assigns different roles women and men through a symbolic and system standards of conduct. The main results showed that the condition of entrepreneur is valued in the community; however it subsumes ethnicity, ie the explicit reproduction of the social and implicitly through the subjectivity linked to religious and secular activities structure. The division of labor in the production of carpets is organized considering the prestige, traditional values and gender roles assignment. Business success is understood as a community success, while there must be a balance between resource use and production of mats, above individual success or single employer.
\end{abstract}

Keywords: division of labour, family enterprises, community, ethnicity. 


\section{Planteamiento del problema}

El proceso de producción artesanal de tapetes de lana de la comunidad de Teotitlán, ubicada en la región de los Valles Centrales de Oaxaca (Oaxaca, México), se organiza con un alto grado de valoración de los elementos étnico-religiosos compartidos en la comunidad. Su traducción del zapoteco es 'lugar cerca de los dioses', o, de acuerdo con los aztecas, 'el Decimotercer Cielo'.

La división vigente del trabajo obedece a una concepción social compartida por los miembros de la comunidad e incluye elementos étnicos y de la tradición zapoteca que comienzan a cambiar. La comunidad es el eje de articulación de las actividades; esta se reconoce como comunidad indígena donde el idioma zapoteco y la tradición de la comida ritual no se han perdido y constituyen elementos que acompañan un estilo de vida que subraya la condición de género (Butler, 1997). Esta condición de género alude a tres elementos: la subjetividad, la relación de identidad en la comunidad y la condición sexo-genérica en el trabajo. Estos elementos pueden observarse en los procesos de producción de tapetes de la comunidad de Teotitlán, donde existe una organización del trabajo basada en el género que comienza a modificarse.

\section{Objeto de estudio}

El objetivo de la presente investigación fue analizar la organización de energía humana en la elaboración de tapetes de las familias de una comunidad indígena de Oaxaca. La organización del trabajo responde a un sistema de género tradicional de asignación de roles distintos para mujeres y varones. Se reproduce gracias a un sistema simbólico y de normas de conducta que asigna funciones y roles a cada sujeto en la comunidad. Se analizan las posibilidades de cambio a raíz de dos elementos: uno relacionado con el contexto externo, es decir, con los cambios socioeconómicos de la región, que enmarca los procesos de migración y la creación y uso de redes de los teotitecos en los Estados Unidos; y otro interno, propio de la dinámica de la comunidad, con la incorporación de las mujeres teotitecas al mercado laboral en la región de los Valles Centrales.

Se estudiaron los procesos económicos relacionados con la elaboración de tapetes de lana. Los procesos económicos se articulan con los procesos políticos y de organización del ciclo ritual anual correspondiente a la tradición católica como mecanismos sociales de poder que refuerzan el sistema binario de género vigente.

Entre los procesos económicos que se involucran en la fiesta religiosa sobresalen la división del trabajo y la asignación por género de las actividades relacionadas con el cuidado y la preparación de las fiestas y el ritual, así como el uso de los recursos naturales. 


\section{Fundamentación teórica}

En relación con la división del trabajo en la comunidad, esta responde a un sistema sexual y de diferenciación sexual organizado con reglas y normas que rigen la conducta de los sujetos (Chodorow, 1984). Las normas se aplican para todas las actividades productivas y de ritual. La organización de la producción de tapetes obedece primariamente a una división sexual del trabajo entre mujeres y varones (sistema binario) que comienza a modificarse con la participación de las mujeres en el mercado laboral, la migración hacia los Estados Unidos y las estrategias de comercialización de los productos artesanales. Estos cambios en la condición de género suponen la construcción de nuevas estrategias y procesos transicionales de género.

Se utiliza la categoría de género como una variable transversal (Lamas, 2007; Burin y Meler, 2010; González, 2013) para comprender los procesos de diferenciación y subordinación en el interior de la comunidad entre mujeres y varones; esta categoría nos permite entender la división de tareas, la asignación de los espacios público/privado a varones y mujeres y el origen de la división sexual del trabajo en la comunidad (Hirata y Kergoat, 1997). Asimismo, se incorporan dos aspectos relevantes relacionados con los dos elementos antes mencionados: el cambio del contexto socioeconómico de la región y el mercado laboral. En relación con el primero, existe una reorientación en la estrategia de venta con el rescate de lo natural aplicado a la elaboración de tapetes; en este sentido, lo natural se entiende como el conjunto de medidas que se incorporan en los procesos de elaboración de los tapetes, desde el cuidado de las ovejas (pastoreo, trasquila) hasta el proceso de obtención del hilo (carda) y su teñido con elementos naturales (añil, cochinilla, flores o nuez, entre otros). El segundo incluye las redes de la comunidad en el exterior, particularmente en los Estados Unidos, para vender los tapetes; el énfasis en las condiciones sustentables de producción puede verse como una estrategia empresarial de venta.

\section{El diseño y la metodología}

Se buscó conocer la percepción de los sujetos y las posibilidades de cambio en relación con la división del trabajo dominante a través de un enfoque metodológico cualitativo; se utilizaron dos métodos: el estudio de caso y la fenomenología. Con respecto al primero, de acuerdo con Giménez (2012), se trata de un caso crítico pues se buscan características tales que permitan confirmar o invalidar una proposición. En el caso de Teotitlán del Valle, es una comunidad indígena que se rige por usos y costumbres y cuyo arraigo religioso (católico) es impor- 
tante. Las familias son extensas y existe entre los teotitecos una alta valoración hacia lo zapoteco y los elementos culturales relacionados con su identidad como zapotecas. El uso del idioma zapoteco también es generalizado y la práctica de ritos y rituales tradicionales se realiza de acuerdo con las normas de la comunidad. El caso crítico permite conocer si los cambios en las estrategias laborales y de migración de algunos de sus habitantes tienen efectos sobre la asignación de los roles de género en el sistema de género dominante. De esta manera se mantienen constantes las variables de etnia y religión. Con respecto a la fenomenología, se presenta la información que se recuperó de entrevistas semiestructuradas de los productores mujeres y varones de la comunidad. La proposición que guía este trabajo es que los cambios culturales se pueden observar en un espacio geográfico-temporal, en la dimensión económica, es decir, en la asignación por género de las actividades que son tradicionales en la comunidad. Asimismo, las condiciones de incorporación de las mujeres a las actividades laborales fuera de la comunidad han coadyuvado a la percepción sobre el papel que estas desempeñan respecto a los emprendimientos y su relación con el éxito/fracaso de estos. El trabajo de campo se realizó en los meses de marzo, mayo, julio y noviembre de 2013 a 2014, e incluyó en una primera fase una revisión bibliográfica y de estadísticas sobre la región para ubicar el contexto y los actores. La población de referencia es de 5.638 personas en el municipio (INEGI, 2010), con una población potencial de 3.600 personas bilingües; la población objetivo de este estudio son familias extensas avecinadas en la cabecera municipal de Teotitlán del Valle que desarrollan actividades relacionadas con el tejido de tapetes, hablan zapoteco y son católicas (50 familias). Se excluyeron los tejedores que trabajan para otros talleres y solo se incluyó un tejedor de una cooperativa. La muestra fue cualitativa, se realizaron 14 entrevistas; la mitad de ellas fueron grabadas $y$, en las que no fue permitido hacerlo, se tomaron notas de campo. Las entrevistas se transcribieron y la información se agrupó en tres dimensiones: migración, actividades económicas y tradición (Creswell, 2013). Se realizaron también entrevistas previas a personas de la familia Mendoza Ruiz: Abigail y Gabriel Mendoza, ambos ampliamente conocidos dentro y fuera de la comunidad. Esta información sirvió para ubicar a los sujetos en un tiempo y en un espacio; el contexto y las actividades cotidianas de los sujetos sirvieron para entender la reproducción ideológico-económica que se expresa en un sistema de género (Giddens, 2011). Se complementó la información con datos y estudios realizados en el Centro de Investigaciones y Estudios Superiores en Antropología Social (CIESAS) de Oaxaca (Robledo, 2009; Dalton, 2010; Hernández-Díaz, 2012). 


\section{Principales resultados}

Se encontró que existe un proceso tradicional en la elaboración de tapetes que enfatiza elementos mercadológicos como el uso de tintes naturales en la lana. Parte de la estrategia empresarial en la producción de tapetes incluye los elementos de apego a la tradición y la búsqueda de nuevos nichos de mercado. En la comercialización de los tapetes se utilizan mecanismos que incluyen la construcción de redes en Oaxaca y en el extranjero, así como un discurso que busca promover la venta de los productos como orgánicos, relacionando elementos tradicionales o ya utilizados por costumbre en la comunidad con requerimientos de nuevos mercados $y$ consumidores, específicamente sobre el cuidado del medio ambiente y productos personalizados (Lipovetsky, 2013). El éxito empresarial se entiende como un éxito de la comunidad por cuanto debe existir un equilibrio entre el uso de los recursos y la producción de tapetes por encima del éxito individual o de un solo empresario. La noción de comunidad está muy arraigada entre los emprendedores de Teotitlán. El apego a la fiesta y a la tradición se observa en el cumplimiento del ciclo ritual religioso cada año, así como en la articulación de los elementos ideológicosimbólicos con los políticos o de cargos (mayordomías) y económicos (Montes del Castillo, 1989). Los resultados se presentan a continuación.

\section{División sexual del trabajo y género. La elaboración de tapetes}

El sistema sexual de organización de la producción es de predominio masculino organizado en torno a dos sexos o de sistema binario. Este sistema de sexo/género (Rubin, 2013) es el resultado de la organización familiar y de las relaciones de parentesco; es moldeado a través de las instituciones y los procesos simbólicos. En Teotitlán, a través de la fiesta religiosa y los rituales. Los procesos económicos se entrelazan estructuralmente con los procesos ideológicos y simbólicos, específicamente con la institucionalización ritual de las fiestas.

En los procesos económicos de emprendimiento puede observarse la división del trabajo y el uso de los recursos naturales, su apropiación y la organización por género. Existen mecanismos comunitarios de uso de la energía humana que se vinculan con los procesos económicos y que tienen como fin la distribución de los recursos entre los miembros de la comunidad (guelaguetza) y el aprovechamiento de la energía individual para obras de tipo comunal (tequio).

La producción de tapetes en Teotitlán del Valle es una tradición familiar; la división del trabajo considera como base la división por género que existe en la comunidad y que establece las responsabilidades diferenciadas para mujeres y varones en las diversas actividades agropecuarias, de transformación y de elabo- 
ración de artesanías. La organización en la producción de los talleres familiares no contradice la organización por género de la comunidad.

La comunidad entera está relacionada con la producción de tapetes, de manera directa en la producción o indirecta en la comercialización; para la venta existen redes en Oaxaca, en México y en el extranjero que evitan en la mayoría de los casos a los intermediarios; muchos teotitecos en el exterior participan en ellas y promueven no solo la artesanía sino también la comida y su estilo de vida. La organización tradicional sobre el uso de la lana, el proceso de trasquila y de carda y la obtención del hilo contrasta con la organización moderna en la venta y colocación de los productos en nuevos nichos de mercado.

Bueno, lo natural es para la gente que consume, que compra, es más apreciado porque tampoco hay que dejar de lado que sí hay este, trabajos, productos industrializados. El mismo... tinte también no es solamente el natural, el natural tiene un costo mayor y también, pues, mucha gente lo produce, la cochinilla. Pero hay otros materiales, por ejemplo, el añil y otras hojas..., se traen de otra región, de la región del Istmo (Gutiérrez, 2014; comunicación personal).

Todo a mano, porque también esto... de lo natural..., hubo un momento en que la demanda del producto artesanal fue tan fuerte..., entonces se recurrió a los productos industrializados... Pero después se vio que la calidad es..., no es la misma y por ello, pues, se empezaron a..., a buscar alternativas o a recuperar lo que, este, lo que anteriormente sí se hacía, porque la cochinilla, pues, era lo que utilizaban las personas (Gutiérrez, 2014; comunicación personal).

En la elaboración de tapetes, todos los miembros de la familia participan por lo menos en alguno de los siguientes procesos: preparar los materiales, cardar, hilar, teñir y tejer. Las familias son extensas, comparten grandes casas organizadas espacialmente alrededor de un centro o patio donde pueden realizarse las actividades relacionadas con el telar. El proceso de elaboración es manual y se aprende desde los cuatro o seis años; normalmente las personas de la tercera edad siguen elaborando tapetes.

En todos los casos los talleres son familiares. En ellos no siempre se produce la lana. Para la elaboración del hilo y del tejido del tapete, existe una división del trabajo. Las mujeres por tradición pueden realizar cualquier actividad, a excepción de los tapetes de gran tamaño por el tipo de telar que se utiliza. En la mayoría de los talleres se elaboran tapetes con diseños tradicionales teotitecos como el diamante zapoteca, las grecas, la siembra o el maíz; en otros casos, los diseños son bajo pedido (pinturas, imágenes, símbolos, etc.). Este tipo de estrategia ha podido salvar la actividad artesanal al incluir un sector de mercado más exigente en cuanto a las normas y los estándares de los materiales así como al diseño (figura 1). 
Figura 1. Diseño de tapete bajo pedido

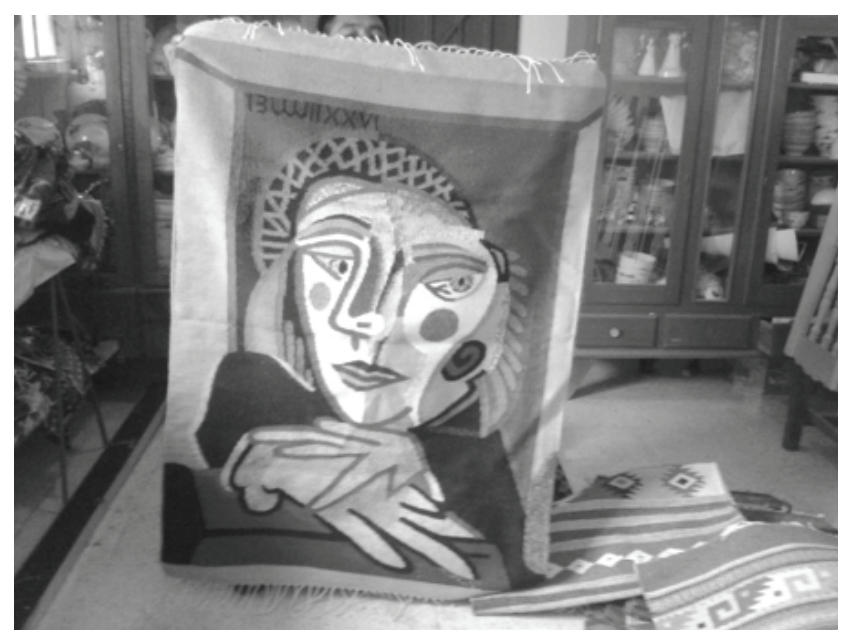

Comúnmente, para la elaboración de un tapete se sigue la tradición en la enseñanza de los procesos: cardar, hilar, entintar, montar el hilo en el telar, señalar el dibujo en los hilos y, por último, tejer. Existe un reconocimiento generalizado dentro de la comunidad para todos aquellos tejedores que han introducido nuevos diseños derivados de los diamantes y ojos. Normalmente se utiliza una técnica para los dibujos geométricos (como grecas) y otra (de calcado) para los diseños con curvas (como peces). Arnulfo Mendoza Ruiz es un pintor que ha introducido nuevos materiales en la elaboración de los tapetes —algodón, seda, oro y plata - y ha recuperado las grecas de Monte Albán y el Diamante de Saltillo; junto con otros artesanos ha popularizado los diseños de milpas, lluvias y montañas y el ojo de Dios. Existen varias cooperativas de producción que agrupan a artesanas y artesanos (Mujeres que tejen sarapes con sus manos, Semillas por la Mujer, Centro de Arte Textil Zapoteco Bii Daüü...); sin embargo, no todas han sido exitosas debido fundamentalmente a las exigencias de la Administración y las responsabilidades de los socios. A pesar de ello, prevalece sobre esta forma de organización de la producción la organización comunitaria, donde los lazos étnico-religiosos y de identidad son más fuertes que el deseo individual de ganancia.

\subsection{Estrategia empresarial en la producción de tapetes}

Para el proceso de elaboración de tapetes y otras artesanías, se sigue la tradición que asigna distintos roles a mujeres y varones en la comunidad; así, los emprendimientos y el proceso de producción quedan en su mayoría a cargo de grupos 
familiares extensos con jefatura familiar de varones. Toda la familia colabora en las diferentes actividades en torno al proceso de elaboración y comercialización: obtención de materia prima, coloración de la lana, manejo de diseños, proceso de promoción y venta. La tradición en la región de los Valles Centrales identifica a cada comunidad con un producto o servicio o, en su caso, con la disposición de materia prima. Así, el trabajo de los tapetes puede rastrearse como el resultado de los procesos de cristianización en la región. La especialización regional está también asociada a los eventos religiosos que se realizan periódicamente y que constituyen una expresión de identidad zapoteca. Las estrategias empresariales que utilizan los teotitecos incorporan elementos del mercado neoliberal: demanda bajo pedido alterna con producción orientada a un mercado específico; innovación y variedad en materiales y diseños; y explotación del concepto natural o sustentable para estimular la demanda (Lipovetsky, 2013). Estas estrategias se despliegan en espacios no necesariamente comunitarios, es decir, los teotitecos establecen redes en la región y fuera de ella — como en los Estados Unidos- para promover y vender sus productos. Estos procesos muestran cambios en los roles tradicionales de género, sin que sean todavía generalizados; son procesos transicionales que intervienen en los procesos de trabajo y emprendimiento.

\section{Proceso de producción}

El proceso de elaboración del tapete (figura 2) comienza con la crianza de las ovejas, de las cuales se obtiene la lana que se comercializará en el mercado municipal de Teotitlán del Valle y sus alrededores. Dependiendo del criador y vendedor, la lana puede entregarse escarmenada, es decir, tras pasar por un proceso donde se despedaza en partes, o limpia y hasta hilada.

La textura del hilo dependerá de la tensión que el productor aplique a cada mechón de lana, además del movimiento de los malacates y las ruedas de madera.

Los hilos pueden ser utilizados en su color natural -en sus variadas escalas de blanco, gris y negro- o ser teñidos en diferentes matices (figura 2; figura 3); para ello, se someten a un proceso que comprende el cocerlos en agua, y se van añadiendo fijativos y colorantes de origen mineral, vegetal o animal. Dependiendo del color deseado, varían los tiempos de cocción, así como la cantidad y variedad de ingredientes que se agregan. El proceso se lleva a cabo en repetidas ocasiones, y, posteriormente, el hilo es lavado y secado. 
Figura 2. El telar

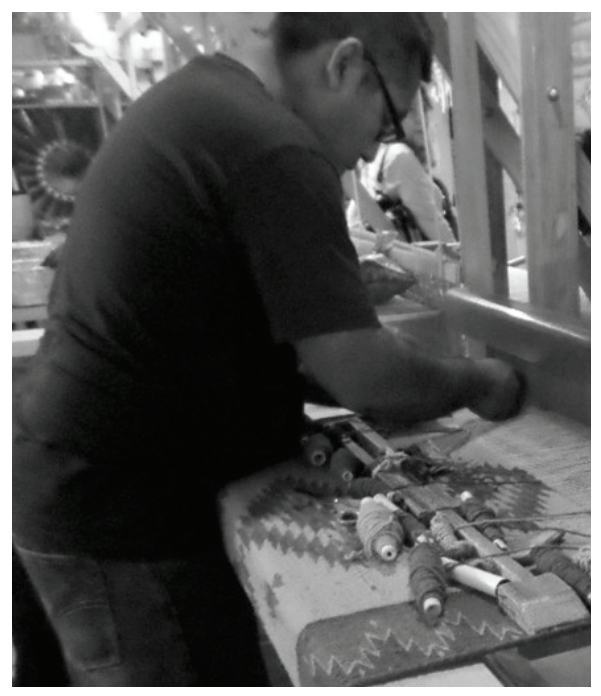

Figura 3, Colorantes naturales.

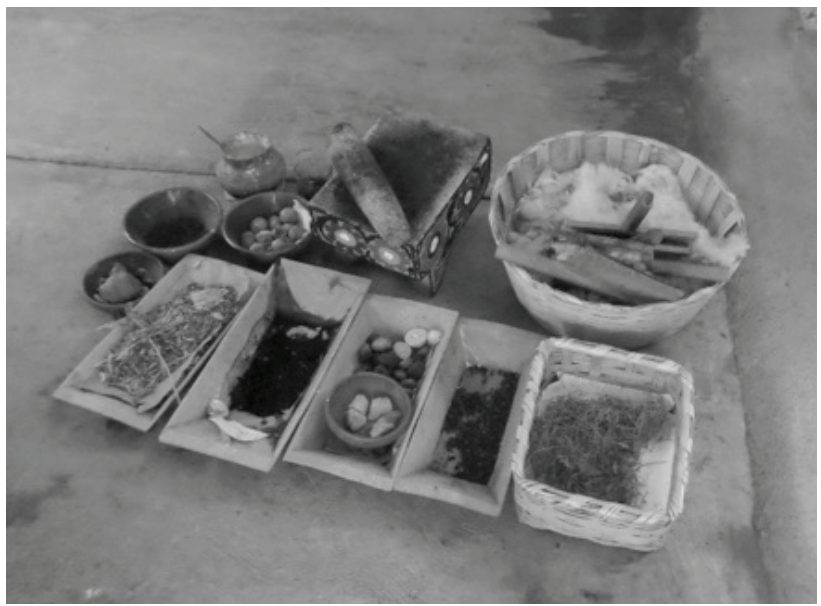

Los teotitecos se enorgullecen por utilizar «productos naturales» para teñir los hilos de lana. Dentro de los colorantes se encuentran la cochinilla de nopal, el añil, el musgo de roca, el huizache, la barba de pirul, el pericón, la granada y la nuez; para fijar los colores, se utiliza lejía de cal, alumbre, jugo de limón, sal natural y vinagre casero, entre otros.

Recientemente los teotitecos han utilizado la condición natural de los materiales de los tapetes y la elaboración manual de estos como parte de una estrategia de mercadotecnia que otorga a los productos el distintivo de orgánicos. Esto les 
ha permitido abarcar nuevos nichos de mercado e incrementar los precios de los productos dado que estos tienen una demanda alta por estar elaborados a mano y estar hechos de diversos materiales (incluidos el algodón y la seda).

\subsection{Organización comunitaria y redes de comercialización}

La tradición étnico-religiosa que existe en la comunidad no desaparece cuando los teotitecos emigran. La tasa de crecimiento poblacional durante el período 2000 a 2005 fue de 0,13 \% (Municipio de Teotitlán del Valle, 2009). En su mayoría los teotitecos emigran hacia los Estados Unidos, aunque algunos residen en Europa. Muchos regresan a la comunidad para participar en las fiestas y los rituales y pagar las mandas a los santos como el Cristo Negro de Esquipulas y la Preciosa Sangre. Algunos también conservan la tradición para la boda y deciden casarse en la comunidad con mujeres teotitecas. Las tradiciones como las fiestas patronales o el Día de Muertos o Navidad constituyen ocasiones especiales para compartir con la familia. La tradición sigue arraigada en los teotitecos aun cuando no compartan los espacios ni la geografía. La razón de ello es el autorreconocimiento como zapotecas, el uso del idioma zapoteco y la adscripción al catolicismo. Los teotitecos en el exterior siguen conservando sus tradiciones y se reúnen y ayudan de acuerdo con la tradición zapoteca. Se realizan tres colectas en la comunidad para las fiestas de la Semana Santa, en honor de la Preciosa Sangre y para las fiestas de la Virgen del Rosario. Aunque los teotitecos en el exterior no puedan asistir a las fiestas, envían el dinero.

En Teotitlán la forma de gobierno son los usos y las costumbres, de manera que existe un apego fuerte a la tradición zapoteca. El pedir guelaguetza o el tequio involucra redes de apoyo y jerarquía entre los miembros de la comunidad. La base de la red es la reciprocidad, entendida como la capacidad de devolver en la misma proporción el bien o servicio que ha sido donado. La equivalencia está dada por los criterios de los miembros de la comunidad en relación con el uso y/o valor de los bienes o servicios y no involucra períodos de tiempo fijos. Así, el pedir guelaguetza para poder emprender un negocio o comenzar una familia aun cuando no se pueda recompensar en el mediano plazo es posible y permite a las familias acumular excedentes. A través de la guelaguetza se conocen el estatus y el poder de cada miembro de la comunidad y su funcionamiento hace posible un sistema de retribución que beneficia a toda la comunidad. Para asegurarse de que esa reciprocidad sea equivalente, cada familia lleva un cuaderno donde se describen la fecha y el tipo de bien o servicio que se recibió o se retribuyó. De esta forma, en las festividades y los rituales es posible seguir la tradición de convidar a comida, pan, fruta y chocolate-atole. 
En relación con las actividades productivas, las redes de apoyo comunitario como el tequio son percibidas en algunos casos como obstáculos para el desarrollo empresarial debido al uso de la energía humana en otras actividades diferentes de las empresariales y el destino comunitario del trabajo. Sin embargo, la identidad étnico-religiosa y la tradición zapoteca siguen predominando en la comunidad. El uso de la energía de los teotitecos tiene un fin comunitario, ya sea que se oriente a las actividades del campo o a las mejoras en la infraestructura de la comunidad o las fiestas religiosas. Ambas formas de uso del trabajo contribuyen a una distribución de la riqueza y a la mejora en las condiciones de vida de los habitantes de la comunidad.

Las redes involucran también aspectos económicos; en relación con la comercialización y venta de tapetes, los teotitecos han logrado explotar un mercado específico enfocado hacia la producción bajo pedido y con materiales naturales. El uso de lana y colorantes naturales, así como la elaboración a mano de los tapetes en diseños tradicionales y modernos, han atraído a un sinnúmero de compradores extranjeros. Otra estrategia de venta es tener puestos fijos en la ciudad de Oaxaca o participar en las ferias itinerantes en otros estados. Estas ferias aprovechan el calendario de fiestas de otras comunidades y las estrategias del gobierno del estado para promover las actividades artesanales.

Para la venta de los productos, los teotitecos se organizan al interior de la familia y entre las familias. Cuando se realiza una venta, se asigna el dinero a la persona que elaboró el tapete, y cuando se conocen oportunidades de venta, las familias de la comunidad establecen quién puede aprovechar mejor la venta. De esta forma, la estrategia de venta tiene como base la comunidad indígena y no el empresario individual, porque el beneficio se concibe comunitario.

\subsection{Identidad zapoteca y mecanismos sociales de poder}

Los procesos que tienen relación con la distribución del poder en Teotitlán son los de nivel ideológico-simbólico, que incluyen los rituales de pasaje, reguladores y periódicos, y los procesos de distribución del poder con los comités religiosos y seculares. Cada individuo en la comunidad tiene asignado un espacio en la estructura y le corresponden ciertas actividades tanto en relación con el ciclo ritual religioso y el secular (en Teotitlán puede decirse que están correlacionados) como respecto a la condición sexo-genérica de los sujetos.

La comunidad organiza su ciclo ritual conforme a lo que marca la tradición religiosa católica; por ello, existen períodos de tiempo especiales para celebrar: la Semana Santa o cuaresma y la Navidad. También están las fechas especiales dedicadas a los santos patrones del pueblo: la Preciosa Sangre, la Virgen de la 
Natividad y la Virgen del Rosario. Hay rituales relacionados con las bodas: velas para contentar y los fandangos (seculares y religiosos). Los rituales más importantes se relacionan con procesos reguladores y periódicos y se corresponden en su mayoría con las ceremonias que acompañan a la fiesta religiosa. Los rituales de pasaje corresponden a la celebración del nacimiento, la confirmación, las bodas y la extremaunción de los miembros de la comunidad; estos involucran el cambio en el estatus de los individuos en la comunidad (Van-Gennep, 1969; Díaz, 1998; Hernández-Díaz, 2012); todos estos rituales se acompañan con música y comida especial.

En los rituales se reproduce la estructura jerárquica que predomina en la comunidad y que se reproduce también en los procesos económicos. El género, la edad, el prestigio adquirido en la comunidad y la jerarquía en la estructura social se respetan tanto en los rituales como en la elección de los comités y en los procesos de producción (figura 4). Ejemplo de esta estructura son las disposiciones del presidente del comité o del huehuete o escribano (Hernández-Díaz, 2012) en las ceremonias seculares o religiosas. La disposición del espacio, los objetos que acompañan al ritual, el lenguaje durante las ceremonias, los turnos de baile, el orden de los invitados..., están rígidamente establecidos y se convierten en norma para los habitantes de Teotitlán.

Las ceremonias religiosas suelen ser de adoración a los santos, de ritos de paso (compromisos matrimoniales, compadrazgos) o de comités para acuerdos comunitarios; todas ellas muestran el estatus de las parejas en la comunidad. La tradición marca que sean parejas católicas casadas con arraigo en la comunidad las que tengan mayor responsabilidad en los ritos comunales. En relación con la división del trabajo para la elaboración de la comida, existen también normas rígidas para las mujeres. La elaboración de la comida ritual involucra no solo el conocimiento que las mujeres tienen sobre los ingredientes y la elaboración a la usanza antigua de la comida ritual, sino que además estas deben tener un reconocimiento entre los miembros de la comunidad. El uso de los elementos naturales como la cera para las velas en la ceremonia de contentar o el uso del cacao patach (cacao blanco, cacao silvestre patasht, pataxtle, patastle de Chiapas) para la elaboración del chocolate-atole, así como de otros ingredientes silvestres prehispánicos, no solo sirve para conservar la tradición, sino que también responde a un uso dentro de un mercado de consumo que prefiere lo natural, lo hecho a mano. Hoy en día las conductas rituales y las prescripciones sobre el uso de instrumentos por género son parte de las estrategias de venta o de atracción turística. Este tipo de prescripción ritual se sigue también en la preparación de otros platillos como el mole (chichilo, negro, coloradito, rojo, amarillo, verde, mancha manteles), la segueza o los higaditos de pollo. 


\section{Figura 4. Ciclo ritual en Teotitlán del Valle}

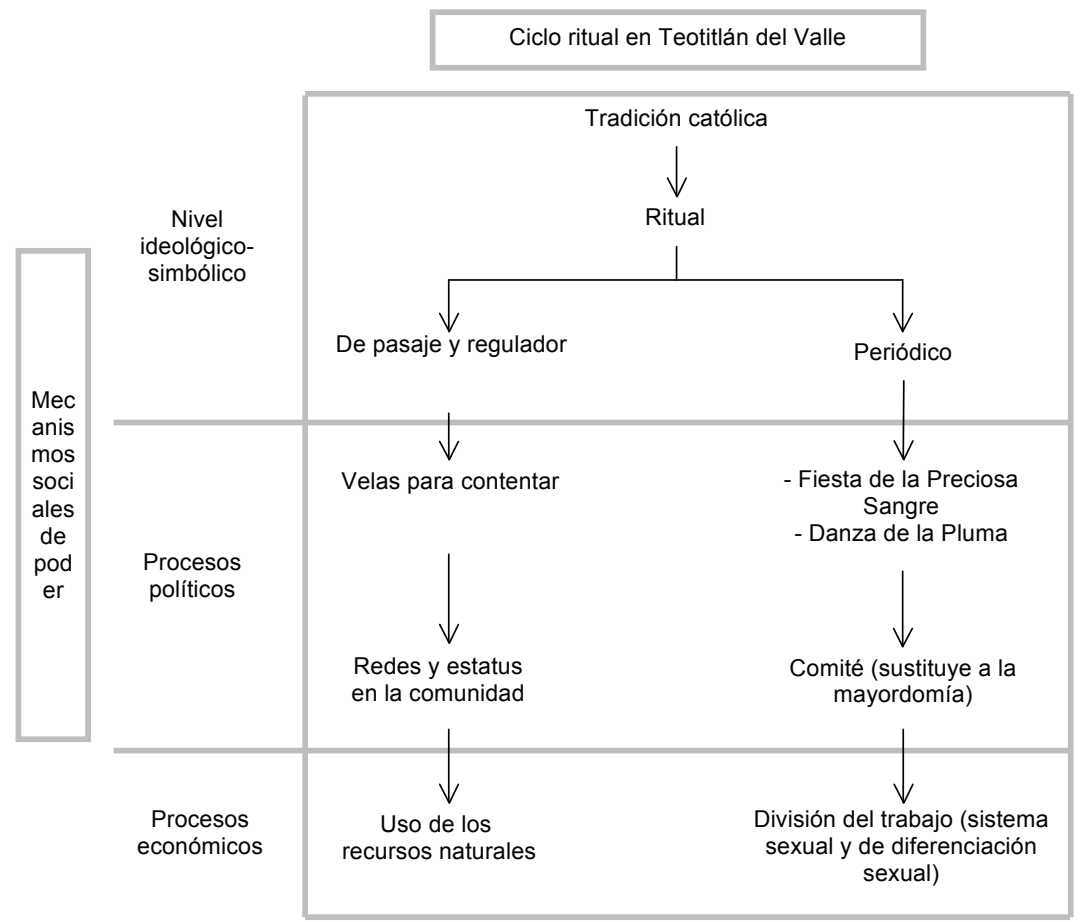

La fiesta tiene un elemento ideológico-simbólico relacionado con la tradición católica; uno de relaciones políticas y redes entre los miembros de la comunidad, que es el medio para ejercer poder o reforzar el estatus de los individuos así como para distribuir los recursos; $y$ un tercer nivel, relativo al proceso económico, que en el caso de la fiesta se refiere a la organización del comité del templo para asegurar que exista continuidad en los festejos. La fiesta religiosa es un evento en el que participan todos los miembros de la comunidad. Es importante porque reúne no solo a los teotitecos que están fuera de la comunidad, sino también a turistas que pueden consumir los productos artesanales. La fiesta religiosa refuerza la identidad de género, étnica y comunal; para llevarla a cabo, se realizan tres colectas en la comunidad, de acuerdo con la importancia de la celebración: para la Semana Santa, para la Preciosa Sangre y para la Virgen del Rosario. La fiesta es la ocasión para visitar la comunidad y establecer redes para la venta fuera de la región.

Para la organización de la fiesta se articulan grupos de hombres que constituyen los comités. Esta figura ha sustituido las antiguas mayordomías, pero mantiene vigente la disposición simbólica y de relaciones de poder que eran parte de esa institución. 
Los miembros del comité son trece personas (varones adultos mayores de 50 años, casados), con estatus reconocido en la comunidad, que tienen a su cargo la organización de las fiestas, la distribución del trabajo y de los bienes suntuarios — que incluyen la comida asignada para cada ritual - y la elaboración de las velas y los ofrecimientos o mandas a los santos entre los miembros de la comunidad (figura 5). Si se analiza el ritual por temporalidad (figura 6), podrá observarse que la mayoría de los rituales son periódicos; los rituales reguladores se corresponden con el invierno y con las fiestas de abundancia como la Natividad, Niño Dios o fiesta de la Cuevita.

Las actividades económicas se entrelazan con las actividades religiosas y las rituales. La actividad de las velas y la elaboración de tapetes son dos ejemplos de ello. Las velas se utilizan en ritos relacionados con los compromisos, el «robo» de la novia y la explicación que se debe dar a los padres al descubrir que su hija no está en casa. El intermediario entre los padres y el novio de la hija ausente es el huehuete. Esta mediación requiere la elaboración de velas aromáticas, cuya luz representa a la hija ausente. El número de velas varía en relación con la prosperidad de los contrayentes; Gagnier (2007) cita un ritual donde fueron necesarias 87 velas para contentar. La elaboración de las velas es manual y, en general, labor de mujeres; se busca cera especial (del Istmo, en algunos casos), aromática, y se utilizan colores naturales como la cochinilla para elaborar flores multicolores de diferente tipo que adornan cirios y velas que pueden llegar a medir hasta dos metros de altura, dependiendo de la importancia y estatus de los contrayentes. Además de las velas, se incluyen fruta y pan en grandes cantidades. Todo esto, a excepción de las velas, se reparte entre los parientes y las amistades que dan fe del compromiso de los futuros novios. Así, comienza un proceso de reciprocidad que culmina con la ceremonia religiosa. Las actividades económicas relacionadas incluyen a miembros de la comunidad, tanto en la elaboración de las velas como en las flores, pan y fruta.

Para la elaboración de tapetes se requiere la energía de toda la familia extensa; la división del trabajo se basa en un sistema sexogenérico binario, pero no hay concentración de los ingresos. Cada miembro adulto recibe lo proporcional a su trabajo.

Los rituales se siguen llevando a cabo en la comunidad a pesar de los cambios en las estrategias laborales por género. La migración no ha impedido que los teotitecos vuelvan o que financien la fiesta desde el exterior. Son los cambios en las actividades económicas los que han comenzado a modificar la percepción sobre el papel que desempeñan las mujeres en la comunidad. 


\section{Figura 5. Ciclo ritual religioso}

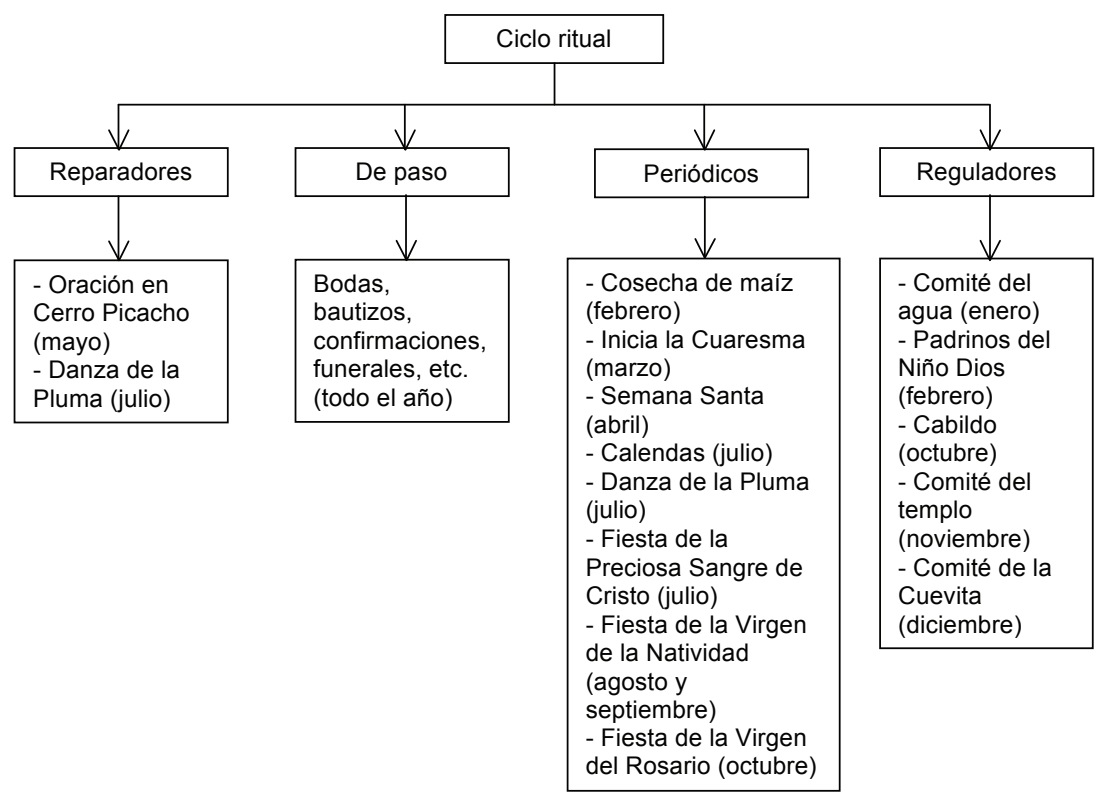

Figura 6. Ciclo ritual religioso por tiempo

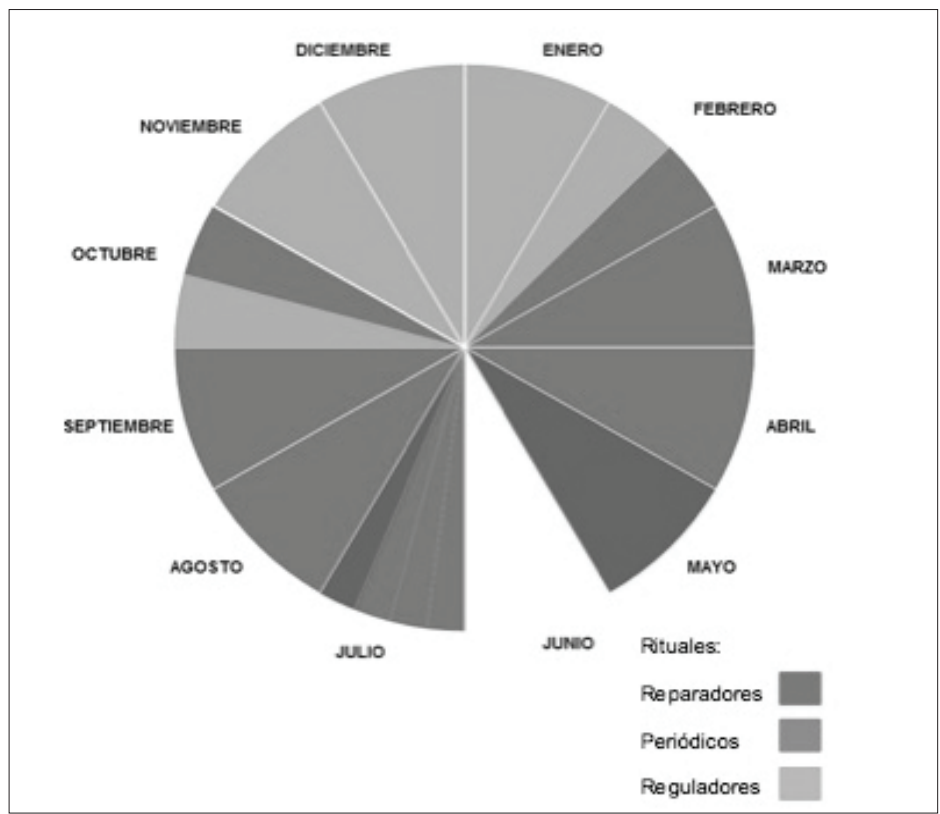




\section{Reflexiones finales}

En la comunidad de Teotitlán existe un sistema de género binario que puede observarse en los procesos económicos y en las actividades seculares y religiosas. El proceso artesanal de producción de tapetes es un ejemplo de asignación sexogenérica del trabajo que no contradice la organización por género que existe en la comunidad. La producción de tapetes involucra una división del trabajo que se apega a la tradición y los roles de las mujeres y los varones. Existen mecanismos comunitarios de uso de la energía humana que se vinculan con los procesos económicos y que tienen como fin la distribución de los recursos entre los miembros de la comunidad y el aprovechamiento de la energía individual. Estos mecanismos se basan en la tradición y apuntan a la estabilidad en los roles del sistema de género dominante y se reproducen gracias a un sistema simbólico y de normas de conducta. Otros elementos que se articulan en la comunidad son el apego a la tradición católica y el autorreconocimiento como zapotecas. Los procesos que tienen relación con la distribución del poder en Teotitlán son los de nivel ideológico-simbólico, que incluyen los rituales de pasaje, reguladores y periódicos, y los procesos de distribución del poder con los comités religiosos y seculares. Sin embargo, es en los procesos económicos donde comienza a observarse el cambio. La estrategia empresarial en la comercialización de tapetes es un elemento de transición al abrir nuevos mercados, explorar el concepto de lo natural o sustentable y establecer redes en la región y fuera de ella para vender los tapetes. Esta estrategia mantiene elementos étnicos de distribución de las ganancias en la comunidad, pero al mismo tiempo abre la posibilidad de cambios a través de las redes con los teotitecos que han migrado a los Estados Unidos. Aun cuando muchos teotitecos conservan sus tradiciones fuera de la comunidad, el cambio en la condición laboral de las mujeres y la migración son procesos transicionales que coadyuvan a cambios en la asignación por género de las actividades que son tradicionales en la comunidad.

\section{Referencias}

Burin, Mabel y Meler, Irene (2010). Género y familia. Poder, amor y sexualidad en la construcción de la subjetividad. Buenos Aires: Paidós.

Butrer, Judith (1997). Lenguaje, poder e identidad. España: Editorial Síntesis. CHodorow, Nancy (1984). El ejercicio de la maternidad: psicoanálisis y sociología de la maternidad y paternidad en la crianza de los hijos. Barcelona: Gedisa.

Creswell, W. John (2013). Qualitative inquiry and research design. Choosing Among Five Approaches. Estados Unidos: SAGE. 
Dalton, Margarita (2010). Mujeres: género e identidad en el Istmo de Tebuantepec. Oaxaca. México: CIESAS.

DíAz, Rodrigo (1998). Archipiélago de rituales. Teorías antropológicas del ritual. México: Anthropos. Universidad Autónoma Metropolitana.

Gagnier, Mary Jane (2007). Rituales de Armonía. Fiestas de Teotitlán del Valle. México: Consejo Nacional para la Cultura y las Artes. Artes de México.

Giddens, Anthony (2011). La constitución de la sociedad. Bases para la teoría de la estructuración. Buenos Aires: Amorrortu.

Giménez, Gilberto (2012). «El problema de la generalización en los estudios de caso». Cultura y representaciones sociales, año 7, núm. 13: 40-62.

González, María Nohemí (2013). ¿Qué hacer con la identidad de Género? ¿Subvertirla, situarla o disolverla? Reflexiones desde la filosofía crítica feminista. Barranquilla: Ediciones Universidad Simón Bolívar.

Hernández-Díaz, Jorge (2012). La Danza de la Pluma en Teotitlán del Valle. Expresión de identidad de una comunidad zapoteca. México: Secretaría de las Culturas y Artes de Oaxaca.

Hirata, Helena y Kergoat, Daniele (1997). La división sexual del trabajo, permanencia y cambio. Argentina: Asociación Trabajo y Sociedad.

INEGI, Instituto Nacional de Estadística y Geografía (2010). México en cifras. Teotitlán del Valle, página web < http://www3.inegi.org.mx/sistemas/Movil/MexicoCifras/mexicoCifras.aspx?em =20546\&i=e.>

Lamas, Marta (2007). Miradas feministas sobre las mexicanas del siglo Xx. México: Fondo de Cultura Económica. Consejo Nacional para la Cultura y las Artes.

Lipovetsky, Gilles (2013). La felicidad paradójica. México: Anagrama.

Montes-del-Castillo, Ángel (1989). Simbolismo y Poder. Un estudio antropológico sobre compadrazgo y priostazgo en una comunidad andina. Madrid: Anthropos.

Municipio de Teotitlán del Valle (2009). Plan Municipal de Desarrollo Teotitlán del Valle «Xaquija», página web < https://www.finanzasoaxaca. gob.mx/pdf/inversion_publica/pmds/08_10/546.pdf. $>$

Robledo, Gabriela Patricia (2009). Identidades femeninas en transformación: religión y género entre la población indígena urbana en el altiplano chiapaneco. México: Publicaciones Casa Chata.

Rubin, Gayle (2013). «El tráfico de mujeres: notas sobre la economía política' del sexo». En Marta Lamas (compiladora). El género: La construcción cultural de la diferencia sexual. México: PUEG.

Van-Gennep, Arnold (1969). Los ritos de paso. España: Antropología Alianza Editorial. 


\section{Agradecimientos}

Este artículo es el resultado de la investigación financiada por la Universidad Autónoma de Querétaro dentro del Fondo para el Fortalecimiento de la Investigación (FOFIUAQ). Participaron en el trabajo de campo y análisis de resultados estudiantes de los programas de verano científico que organizan la Academia Mexicana de Ciencias y el Programa Delfín: Martha Yamileth Nieto García, del Instituto Tecnológico de Tepic, y Carlos Alberto Castillo Tavera, de la Universidad Autónoma de Querétaro. 